Revue d'histoire de l'Amérique française

ZRS REVUE D.HISTOIRE DE L'AMÉRIQUE FRANÇAISE

\title{
Le père Pierre-Joseph-Marie Chaumonot, missionnaire de la Huronie (2ème partie)
}

\section{André Surprenant}

Volume 7, numéro 2, septembre 1953

URI : https://id.erudit.org/iderudit/301594ar

DOI : https://doi.org/10.7202/301594ar

Aller au sommaire du numéro

Éditeur(s)

Institut d'histoire de l'Amérique française

ISSN

0035-2357 (imprimé)

1492-1383 (numérique)

Découvrir la revue

Citer cet article

Surprenant, A. (1953). Le père Pierre-Joseph-Marie Chaumonot, missionnaire de la Huronie (2ème partie). Revue d'histoire de l'Amérique française, 7(2),

241-258. https://doi.org/10.7202/301594ar d'utilisation que vous pouvez consulter en ligne.

https://apropos.erudit.org/fr/usagers/politique-dutilisation/ 


\title{
LE PERE PIERRE-JOSEPH-MARIE CHAUMONOT, MISSIONNAIRE DE LA HURONIE
}

\author{
$(\text { suite })^{*}$
}

\section{CHAPITRE II}

\section{APPRENTISSAGE}

D'après l'autobiographie, il semble que le 3 août soit la journée du départ de Québec ${ }^{1}$. Plus tard, Chaumonot écrira au Père Nappi: "La veille de la fête de saint Laurent, je m'embarquai dans un canot de sauvages hurons (c'est le nom de ce peuple) sur la grande rivière du glorieux martyr"'. Ce 9 août serait donc la date d'un second départ, probablement des Trois-Rivières, "qui était le lieu de ralliement naturel pour les Algonquins et les Hurons de l'Ouest, pour les Algonquins du Saint-Maurice et ceux du bassin laurentien.",3

Le cinquième jour du voyage, les PP. Poncet et Chaumonot durent se séparer et voyager dans des embarcations différentes; "cependant les canots voyageaient de compagnie de sorte que presque tous les soirs, ils se retrouvaient pour souper et passer la nuit". "Nous avions même très souvent la facilité de célébrer la sainte messe le lendemain matin avant le départ. Ce fut la seule consolation de tout le voyage, qui fut de trente jours pour moi et de trente-deux pour le Père Poncet4."

Cette messe, célébrée à l'aube, était réellement le seul souti en des missionnaires au milieu des difficultés inouies d'une telle marche.

* Voir Revue d'Histoire de l'Amérique Française, juin 1953: 64-87.

1. $\mathrm{AB}, \mathrm{I}: 50$.

2. Chaumonot à Philippe Nappi, le 1er mai 1640, Martin, 48.

3. Latourelle, I: 49.

4. Chaumonot à Nappi, le 1er mai 1640, Martin, 49. 
Chaumonot, dans une lettre, bien précieuse pour nous, adressée au Père Nappi de Rome, a raconté avec sa simplicité coutumière son voyage en Huronie $^{5}$ :

Je n'ai done pas à raconter à V.R. que ce qui a été la cause de nos souffrances. Il y en avait beaucoup: l'air, l'eau, la terre, le canot, la nourriture nouvelle pour nous, la grossièreté des sauvages et l'ignorance de leur langue.

L'air nous faisait souffrir tantôt par sa chaleur excessive, tantôt par le froid, tantôt par les vents, les brouillards, la pluie et quelquefois la grêle. C'est une chose vraiment étrange que dans le mois d'août et au commencement de septembre, nous ayons eu cette variété de température. Je ne puis l'attribuer qu'au nombre incroyable de lacs et de forêts qui couvrent le sol et qui dégagen ‘ tant de vapeurs que, après une journée brûlante, nous avions souvent des journées glaciales avec pluie, grêle et brouillards.

L'air en outre est rempli pendant l'été d'une si grande quantité de moustiques très affamés, qu'il nous semblait être en Egypte, subissant le châtiment que Dieu infligea à Pharaon... Ils nous tourmentaient surtout le soir, quand nous débarquions: car sur l'eau ils paraissaient moins pendant le jour. Leur piqûre produit une tumeur rouge qui rend quelque fois la peau semblable à celle d'un lépreux.

L'eau nous menaçait de mort presque chaque jour, et par ses tempêtes, et par sa rapidité, et par ses cascades, et par les torrents qu'il fallait franchir, le moment le plus pénible (et il se renouvelait chaque jour) était soit lorsque la rivière que nous suivions se trouvait remplie de rochers, au milieu desquels notre canot ne pouvait pas avancer, soit lorsque nous arrivions à l'extrémité du lac que nous traversions. Pour passer d'un lac dans un autre ou d'une rivière dans une autre, il fallait décharger tout le bagage sur le rivage, et le porter à dos jusqu'à l'endroit navigable d'une autre rivière ou d'un autre lac, à des distances quelquefois d'une ou deux lieues, sans chemin tracé et au milieu des épines et des bois, des montagnes et des précipices. J'ai vu quelquefois mon pauvre compagnon gémir sous la lourde charge de son autel portatif, de ses livres et autres objets. Il était baigné de sueurs en même temps que de l'eau de la rivière dans laquelle il nous fallait

5. Voir aussi l'excellent résumé du voyage au Pays des Hurons, fait par le Père Latourelle dans Etude sur les écrits de Jean de Brêbeuf, première partie, Routier de la Huronie, 47-72. 
descendre pour aborder, en marchant sur des pierres quelquefois si aigües qu'elles nous coupaient la plante des pieds, ou si glissantes que nous avions de la peine à nous tenir debout. Ajoutez à cela que nous devions courir à la suite des sauvages qui, habitués à ce travail, marchaient avec une grosse charge au milieu des roches et des épines, et sur des côtes escarpées, comme s'ils avaient été dans une prairie verdoyante. ...Le danger à craindre en s'écartant du chemin n'est rien moins que de risquer sa vie, car dans ces solitudes il n'y a aucun sentier qui conduise à quelque bourg, ville ou village voisin...

Nous trouvâmes un jour un très grand marécage, couvert d'herbes et d'arbustes qui lui donnaient l'aspect d'une riante prairie, mais en dessous l'eau dormait. Nos sauvages le traversèrent si lestement que nous ne pouvions pas reconnaître la trace de leurs pas sur le terrain plus ferme qu'ils avaient choisi pour marcher avec assurance. Nous les suivions de notre mieux, mais à chaque pas il nous semblait que la terre allait nous manquer, et que nous tombions dans un précipice. Je n'ai jamais vu la terre trembler autant qu'à chaque mouvement de nos pieds.

Pour le canot, il donnait plus à souffrir que tout le reste, parce que nous y restions plus constamment. Ces canots, faits d'écorce, ont environ quinze pieds de long sur trois et quatre de large au milieu. Ils n'ont ni voile, ni tente pour garantir les voyageurs des brûlants rayons du soleil ou des intempéries de l'air. On n'ose pas remuer: le moindre mouvement le ferait chavirer, et jetterait à l'eau tout ce qu'il contient. Il n'est pas permis de s'y tenir debout, ni assis ni un peu haut. On y doit rester toujours replié comme un hérisson. Joignez à cela la malpropreté des sauvages qui répandent une odeur insupportable, et qu'on ne peut éviter.

La nourriture ne consistait que dans un peu de blé d'Inde, nommé en Italie blé de Turquie (si j'ai bonne mémoire), cuit à l'eau pure, sans sel ni aucun assaisonnement. Nous n'avions pour lit que la terre nue ou quelque rocher, et sans aucun abri que la voûte des cieux.

La grossièreté des sauvages ne diffère guère de celle de bien des brutes. Ils n'ont pour vêtement qu'une loque qui couvre les parties honteuses. Dans les grands froids, ils se couvrent d'une peau de cerf ou d'autre bête semblable. Ils ne riaient jamais autant que quand ils nous voyaient dans quelque embarras. Ils sont tellement intéressés qu'ils ne rendent pas le plus petit service sans avoir le prix dans la main; mais la plus grande difficulté venait de ce que nous ne pou- 
vions comprendre ni leurs paroles ni leurs gestes, qui diffèrent des nôtres autant que leur langage...

Malgré tout cela, nous voilà arrivés, grâce à Dieu, à notre destination, pleins de force et de bonne volonté, pour consacrer nos vies au service de Celui qui nous a donné la sienne ${ }^{6}$.

Le canot de Chaumonot, conduit par six sauvages, aborda au lac Isiaragui (Mud Lake) le dix septembre. "Au même moment de notre arrivée, écrit Chaumonot, le Père Jérôme Lalemant, Supérieur de cette mission sentit frémir sa poitrine, ce qu'il prit pour un avertissement que quelqu'un de nos pères de France arrivoit. Il sort aussitôt de la cabane, jette les yeux vers le lac et me voit déjà débarqué au bord de l'eau. Il accourt à moi, m'embrasse avec tendresse et il me mène au logis en me racontant ce qui l'avoit excité à venir à ma rencontre?"."

Outre le Père Lalemant, Chaumonot trouva dix Pères distribués en trois résidences ${ }^{8}$, soit les PP. François Le Mercier, Pierre Pijart, Jean de Brébeuf, Pierre Chastelain, Paul Ragueneau, François du Péron, Simon le Moyne, Antoine Daniel, Charles Garnier, Isaac Jogues'.

Ces apôtres incomparables n'étaient pas les "saints idiots ou les membres compromis qui avaient fait glissade" décrits par Michelet et, encore moins, des hommes que "la nature avait maltraités, ne leur donnant qu'une mine chétive et de la piété", et qui "étaient envoyés dans les missions pour s'y faire griller, rôtir et martyriser, au profit et à la plus grande gloire de l'Ordre ${ }^{10 "}$, comme Voltaire l'affirme, un jour qu'il court après l'esprit. Mais il faut plutôt convenir avec le Père de Rochemonteix: "L'histoire en main, il serait difficile de trouver dans les missions si belles du XVII ${ }^{e}$ et du XVIII ${ }^{\circ}$

6. Chaumonot à Nappi, le 1er mai 1640, Martin, 49-54.

7. $\mathrm{AB}, \mathrm{I}: 50$.

8. Chaumonot à Nappi dans Martin, 55.

9. Voir les recherches de: Arthur Jones, Old Huronia. Fifth Report of the Bureau of Archives for the Province of Ontario (Toronto, 1909), 310-318. A l'avenir, l'abréviation sera: Jones.

10. Carayon, IX. 
siècle une collection d'apôtres comme ceux de la mission huronne" ${ }^{11}$. En 1649, dans une lettre au Père Général de la Compagnie, Vincent Carafa, le Père Ragueneau décrira, d'une façon très élogiєuse, ces illustres collaborateurs de Chaumonot: "L'état de notre maison et même de tout la mission est tel, que je ne crois pas qu'on puisse rien ajouter à la piété, à l'obéissance, à l'humilité, à la patience, à la charité, à la parfaite régularité de tous les nôtres. Nous n'avons tous véritablement qu'un même cœur, une même âme et un même esprit... En quelque manière que Dieu veuille disposer de nous, soit pour la vie, soit pour la mort, ce sera là notre consolation d'appartenir au Seigneur et de lui appartenir pour toujours"12. C'est au milieu de tels missionnaires que devait travailler Chaumonot, au pays des Hurons.

Cette région, occupée par les Hurons, était située, selon le Père Bressani, "entre le 44e et le $45 \mathrm{e}$ degré de latitude. En longitude, il est à trois quarts d'heure à l'Ouest de Québec, (il écrira plus tard " 35 ' seulement de distance horaire entre Québec et les Hurons") et à plus de six heures entières de Rome". Au sud-Ouest, il y a un lac de 1,200 milles environ de circonférence appelé Mer douce (lac Huron). On y voit le flux et le reflux, chose rare ailleurs que dans la mer. Ses îles sont très nombreuses; une d'elles, qui a 200 milles de circuit, est habitée par des Sauvages nommés Outaouais ${ }^{13}$." Ces Hurons sont entourés, "au couchant" par la nation du Petun, "au sud, en tournant un peu vers l'occident", par la Nation Neutre et aussi par la nation du Chat qui habite "sur les rives méridionales du lac Erié", enfin au nord par les nations Algonquines ${ }^{14}$. En somme un tout petit pays qui, au dire de Brébeuf, peut se traverser dans sa plus longue étendue en trois ou quatre jours ${ }^{15}$. Le Père Jérôme Lale-

11. Rochemonteix, 1: 432 .

12. Ibid., 1: 435. Ragueneau lui-môme n'était pas un ouvrier ordinaire. Brébeuf écrira: "Nous avons ici d'excellents religieux et fort capables, mais ils sont inférieurs au P. Ragueneau, surtout pour le gouvernement", Ibid., 1: 435.

13. Félix Martin, éd., et trad. Relation abrégée de Quelques Missions des Pères de la Compagnie de Jésus dans la Nouvelle-France par le Père François-Joseph Bressani de la même Compagnie. (Montréal, 1877), 11. Désormais, l'abréviation sera: RA.

14. RA, 11-14.

15. JR, 8: 114 . 
mant, en 1638, donne au pays un maximum de vingt à vingt-cinq lieues de longueur sur sept ou huit de largeur ${ }^{16}$.

Les premiers Français qui rencontrèrent ces sauvages les nommèrent Hurons "à cause de leurs cheveux droits comme les soies du sanglier, sur le milieu de la tête, ce qu'on appelle en français une hure ${ }^{17}$."

Le Père Jérôme Lalemant nous donne le chiffre de la population de la Huronie au moment de l'arrivée du Père Chaumonot: "Il se trouve dans ces cinq missions trente-deux tant bourgs que bourgades, qui comprennent en tout enuiron sept cens cabanes, de feux enuiron deux milles et enuiron douze mille personnes ${ }^{18}$."

Afin de mieux connaître le milieu où le Père Chaumonot a travaillé, voici quelques notes sur la nourriture, le vêtement, l'habitation et le caractère des Hurons, d'après la Relation du Père François-Joseph Bressani ${ }^{19}$.

Ces Hurons se nourrissent de blé d'Inde, de courges et de haricots, de poissons et de viandes fraîches ou fumées, produits de leurs chasses. Ils ont quelques fruits, citrouilles, mûres, fraises, noix, cerises "très aigres", bleuets ${ }^{20}$. Ils n'ont ni pain, ni vin, ni sel, ni viandes, ni légumes ni autre nourriture commune avec l'Europe ${ }^{21}$.

16. Latourelle, 1: 84. "The region then inhabited by the three great groups, the Hurons proper, the Petuns and the Neutrals, lay entirely within the confines of the present Province of Ontario, in the Dominion of Canada, with the exception of three or four Neutral villages which stood as outposts beyond the Niagara River in New-York State, but which eventually were forced to witdraw, not being backed by the rest of the nation against the Senecas in their efforts to resist the encroachments of the latter. Huronia proper occupied but a portion of Simcoe country, or to be more precised, the present townships of Tiny, Tay, Flos, Medonte, Orillia and Oro, a very restricted territory and, roughly speaking, comprised between $44 \mathrm{deg}$. $20 \mathrm{~min}$. and $44 \mathrm{deg} .53 \mathrm{~min}$. north latitude, and from east to west between $79 \mathrm{deg}$. $20 \mathrm{~min}$. and $80 \mathrm{deg} .10 \mathrm{~min}$. longitude west of Greenwhich", Jones, 422.

17. RA, 19. Voir aussi Q, 1 (1639): 50. Ils ont une grande horreur pour les cheveux frisés, qui sont tout-à-fait rares parmi eux, RA, 19.

18. Q, 1 (1640): 62. Brébeuf, Sagard, Champlain s'accordent pour donner une population d'environ 30,000 âmes. La diminution est due à la petite vérole, mais aussi au fait que ces derniers donnaient un chiffre approximatif (et il est très difficile d'évaluer une population), alors que le Père Lalemant a opéré un vrai recensement. Sur la population des Hurons, voir Latourelle, 1: 84-85.

19. Sur la Huronie, voir l'étude du P. Latourelle, deuxième partie, Brébeuf ethnologue, chapitre premier, Du milieu à l'homme, 1: 83-105.

20. Chaumonot à Nappi dans Martin, 54. Voir aussi RA, 15.

21. RA, 16 . 
Pour les hommes, la manière de se vêtir est libre, mais, à l'exception d'une certaine nation d'Algonquins, tous se couvrent, du moins autant que l'exige la décence. Les femmes sont beaucoup plus modestes. Les Huronnes, même dans leurs cabanes, ont toujours un vêtement qui descend de la ceinture jusqu'aux genoux ${ }^{22}$.

Comme habitation, les Hurons n'ont que des cabanes entourées d'enceintes ou de forts faits avec des pieux entrecroisés et appuyés contre des trones d'arbre ${ }^{23}$. "Leurs cabanes ont 10, 15, 20, 30 et 40 cannes ${ }^{24}$ de long, et consistent en de grosses écorces soutenues par des pièces de bois, qui servent aussi à porter le grain qu'ils font sécher pendant l'hiver. Les uns n'ont pour lit que quelques branches, et les autres quelques écorces ou des nattes. On ne voit dans ces cabanes ni table, ni banc, ni rien de semblable; la terre ou une écorce tient lieu de tout espèce de meubles" ${ }^{25}$. Le Père Bressani ajoute à la suite de cette affirmation: "C'était la nourriture et le logement de nos Pères dans ces missions, regardées à juste titre, comme les plus pénibles de notre Compagnie ${ }^{26}$."

En effet, se faisant "tout à tous", comme l'Apôtre, Hurons avec les Hurons, les Pères adoptaient les coutumes de leurs nouveaux pays; leur façon de vivre "estant en tout semblable à celle des sauuages" ${ }^{27}$. Chaumonot nous donne un admirable témoignage de ce fait dans cette lettre, déjà mentionnée, au Père Nappi:

Nos demeures sont d'écorce, comme celles des sauvages, sans divisions en salle, chambre, cuisine, cave, excepté pour la chapelle. Le reste est en commun. Nous n'avons ni tables, ni banes pour prendre les repas, ni vases ou verres pour boire le vin, l'eau ou autre liquide, ni écuelles, ni assiettes, ni entremets, ni dessert, ni portions, ni pain, ni fruits. Tous les ustensiles de notre cuisine et de notre réfectoire consistent dans

22. RA, 17.

23. RA, 18.

24. La canne, mesure d'Italie, équivaut environ à six pieds, RA, 18.

25. RA, 18.

26. Ibid., 18, "Cette mission était très pénible et il n'y en a jamais eu de semblable. Je ne sache pas en effet que des prédicateurs de la foi dans ces pays étrangers aient été faire un établissement stable à une si grande distance de la mer, avec l'impossibilité de recourir à l'Europe pour la nourriture, le vêtement et tous les autres besoins de la vie", RA, 57.

27. Q, (1639): 57 . 
un grand plat d'écorce, rempli d'une certaine bouillie de farine, de blé d'Inde, cuite avec quelques poissons séchés à la fumée. Voilà tout le sel qu'on y met. Je ne vois rien à quoi l'on puisse mieux comparer cette sagamité (c'est le nom que nous lui donnons), qu'à la colle qui sert à fixer le papier sur les murs.

La soif ne se fait presque jamais sentir, parce que nous ne mangeons rien de salé, et que la nourriture est toujours très liquide. Depuis que je suis ici, c'est-à-dire déjà depuis huit mois, je n'ai pas bu en tout la valeur d'un verre d'eau.

Notre lit se compose d'une écorce et d'une natte épaisse à peu près comme une piastre de Florence. Pour les draps, on n'en parle pas, même pour les malades.

Ce qui nous incommode le plus est la fumée qui, faute de cheminée, remplit toute la cabane, et gâte tout ce que nous voudrions garder. Quand certains vents soufflent, la position est très p énible à cause de la douleur des yeux. Nous n'avons pas le soir, en hiver, d'autre lumière que celle du foyer, pour réciter notre bréviaire, étudier les préceptes de la langue ou autres travaux. Le jour, la lumière nous vient par l'ouverture pratiquée au sommet de la cabane, qui sert en même temps de cheminée et de fenêtre.

Voilà notre manière de vivre dans notre résidence. Quant à celle que nous gardons quand nous allons en mission, V.R. saura d'abord que les sauvages, qui pratiquent entre eux certaines règles de l'hospitalité, n'en tiennent guère compte avec nous. Ainsi nous sommes obligés d'avoir de petits couteaux, des alènes, des bagues, des aiguilles, des pendants d'oreilles et choses semblables, pour payer nos hôtes. Nous portons en outre une couverture pour nous protéger le jour et nous envelopper la nuit...29

Les Hurons n'ont pas la peau très brune, surtout quand ils sont jeunes. Ils sont robustes, d'une taille haute et bien proportionnée. Leur santé est excellente, et ils ignorent jusqu'au nom de bien des maladies communes en Europe, comme la pierre, la goutte, la rupture, etc. On ne rencontre parmi eux ni bossus, ni nains, ni gens de corpulence monstrueuse, ni goîtres etc. Ils sont hospitaliers les uns envers les autres et se visitent très souvent. Ils tiennent à être regardés comme généreux et désintéressés. Leurs sens sont d'une perfection extraordinaire et leur force d'âme pour supporter toute espèce de

28. Martin, 55-57. 
misère est admirable. Ils ont de l'intelligence, raisonnent bien, et sont servis par une mémoire très fidèle. Grâce à un talent oratoire très remarquable, ils traitent un sujet, qu'ils connaissent bien par ailleurs, avec une habileté égale à celle des Européens ${ }^{29}$.

"C'est là, écrit le Père Lalemant, où premièrement on a tourné la teste, où on a esté à l'attaque: et jamais pour quelque aduis, menaces, ou mauuais traittement que le diable ait pû susciter, on n'a quitté aucun dessein, n'y perdu aucune occasion de seruir le maîstre qui nous employe ${ }^{30}$."

Mais, à son arrivée comme Supérieur de toute la mission, le Père Lalemant effectua un changement important: "il préféra à la voie des résidences, prônées jusque là par Brébeuf, la voie des missions: la voie des résidences, dit-il, nous ayant paru pleine d'inconvénients \& bien moins efficace, nous nous sommes résolus à celles des missions, quoy que plus faschtuse de beaucoup \& plus pénible, surtout en ces contrées" "31. Le Père Lalemant supprima donc les résidences de Téanaostaiae et d'Ossossané, qu'il fonda en une unique maison, celle de Sainte-Marie. La construction, entreprise à l'été de 1639, fut achevée "sur le milieu de l'automne". Cette nouvelle fondation ${ }^{32}$ servait à la fois de centre d'opération, d'hôtellerie, d'école, d'hôpital et même de retraite pour les sauvages. Les missionnaires, groupés en équipes volantes de deux, envahissaient le territoire des Hurons, des Neutres, des Pétuns et des Népissings, tandis que quelques autres missionnaires demeuraient à l'année à la résidence, pour servir auprès des malades, des visiteurs, et surtout auprès des catéchumènes qui achevaient leur instruction. Ils

29. Résumé fait d'après Bressani, RA, 23-25.

30. Q, I (1640): 62 .

31. Latourelle, 1: 81. Le Père Lalemant établit donc une résidence centrale, d'où les missionnaires partaient pour les villages des sauvages. Du temps de Brébeuf, les missionnaires fondaient des maisons dans chaque village évangélisé, alors que sous le règne de Lalemant c'étaient simplement des camps volants.

32. "Ce lieu est situé au milieu du pays, sur la coste d'une belle rivière, qui n'ayant pas de longueur plus d'un quart de lieue, ioinct ensemble deux lacs, l'un qui descend à l'Occident, tirant un peu vers le Septentrion, qui pourroit passer pour une mer douce, l'autre qui est vers le Midi, dont le contour n'a guere moins de deux lieues," Q, 1 (1640): 63. La résidence était donc située sur les bords de la rivière Wye, et un peu au sud-est de Midland. 
s'absentaient cependant quelquefois, pour instruire les adultes et baptiser les petits enfants moribonds des villages voisins ${ }^{33}$.

C'est ainsi que les Pères du Perron ${ }^{34}$ et Chaumonot furent choisis pour aider le Père Raguentau ${ }^{35}$ qui reçut le soin principal de l'ancienne résidence devenue "Mission de la Conception."

Chaumonot connut des débuts très difficiles, car les bourgs de la mission étaient infestés d'une terrible maladie contagieuse "une espèce de petite vérole" ${ }^{36}$. Une religieuse de l'Hôtel-Dieu nous décrit cette maladie avec un réalisme presque brutal: "Comme c'était la petite vérole, qui est une maladie fort sale, et que les Sauvages n'avaient point de linge, ils étaient très infects, et cette corruption les avait tous pourris. Il se forma aussi des ulcères et des chancres en leur corps en si grande quantité qu'on ne savait par où les prendre...." Initiation fort pénible pour Chaumonot qui écrira: "Nous ne manquâmes pas tous deux d'occasion d'exercer la patience en exerçant notre $\epsilon$ mploi qui étoit de consoler, d'instruire, de baptiser et d'assister de notre mieux ces pauvres moribonds" ${ }^{38}$. Le Père Lalemant, par son témoignage, confirme ces paroles:

Le Père Paul Ragueneau a eu le soin principal de cette mission; le Père du Perron et le Père Chaumonot l'ont assisté l'un après l'autre, et tous trois n'ont pas eu peu à souffrir et à trauailler, tant pour l'estendue de leur département, que pour la qualité des personnes qui s'y rencontrent: car ayant tousiours habité parmy eux depuis que nous sommes dans le pays, ils se trouuent pour la plupart battus et rebattus de nos mystères et par le mespris qu'ils ont fait de la grâce de Dieu, leur cœur est endurey, et tous les iours de plus en plus ils se vont aigrissant contre luy, à mesure que cette main paternelle les va chastiant pour les réduire à leur deuoir. C'est de là que sont venus les plus mauvais bruits et les plus pernicieux desseins contre nous; ce sont ceux-là qui dans les con-

33. Voir Latourelle, 1: 181.

34. François du Perron est né a Lyon le 26 janvier 1610 et arriva au pays le 30 juin 1638. Il mourut a Chambly le 10 novembre 1665, Melançon, 29.

35. Paul Ragueneau est né à Paris, le 18 mars 1608, et il arriva à Québec le 28 juin 1636. Il revint a Paris, le 12 aoút 1662, où il mourut, le 3 septembre 1680, Melançon, 65 .

36. $\mathrm{AB}, 1: 51$.

37. ES, 2: 389, n. 7 .

38. $\mathrm{AB}, 1: 51$. 
seils publics crioient le plus fort au massacre, et qui ont rempli de calomnies contre nous les nations où nous auons esté de nouueau annoncer l'Euangile, lesquels ils ont sollicitées de nous mettre à mort, pour se pouuoir deffaire de nous auec moins de conséquence ${ }^{39}$.

Cependant, les Pères (13) et les Français (14), répandus dans les bourgs n'étant pas atteints ${ }^{40}$, les Sauvages crièrent rapidement à la sorcellerie. Le Père Laltmant les excusera ainsi: "Il est arriué trèssouvent, et on l'a remarqué plus de cent fois, qu'où nous estions les mieux venus, où nous baptisions le plus de monde, c'estoit là en effect où on se mouroit dauantage; et au contraire dans les cabanes dont on nous deffendoit l'entrée, quoy qu'ils fussent quelquefois malades à l'extrémité, on voyoit au bout de quelques iours tout le monde heuresement guery" ${ }^{41}$. Le Père Ragueneau cite un exemple typique de ce fait: Au bourg de Saint-François-Xavier, il trouve trois frères malades, qu'il instruit de la foi chrétienne. Mais leur mère s'oppose à la réception du baptême, parce que, déjà, elle a perdu un de ses fils, qui avait été baptisé l'été précédent. Le Père Ragueneau laisse la mère blasphémer et prêche aux fils sur les flammes de l'enfer; il s'adresse ensuite à l'aîné, âgé de vingt ans: "Es-tu résolu à ces peines"? "Hélas nenny! baptise moi", rêpond le jeune malade. "Quoi malheureux, s'écrie la mère, es-tu donc résolu de mourir? tu es mort si on te baptise". "Je veux qu'on me baptise, reprend le fils, car je redoute trop ces flammes qui brulent tout. et ne finissent jamais". Le Père Ragueneau lui confère le baptême, tandis que les deux autres n'ont pas assez de courage pour "désobéir sainctement" à leur mère. Huit jours après, Ragueneau retourne dans. cette cabane. Le nouveau baptisé avait été emporté immédiatement. par la maladie, tandis que les deux autres étaient sains et saufs. On s'imagine alors la réception. Cette pauvre mère, écrit Ragueneau,

39. Q, 1 (1640): 78.

40. Excepté Robert le Coq qui fut atteint de la petite vérole et dut partir pour Québec. Abandonné en chemin par les sauvages, il arriva enfin à la maison des Pères Jésuites. Il guérit, il retourna en Huronie pour montrer qu'il était bien vivant et surtout pour démentir ceux qui l'avaient accusé d'avoir dit que les Jésuites étaient les seuls "autheurs" et la seule cause de toutes les maladies. Le récit des malheurs de ce Français se trouve dans Q, 1 (1640): 56-60.

41. Q, 1 (1640): 55. Lire dans ce deuxième chapitre les persécutions subies par les Pères, 55-60. 
n'avait-elle pas quelque raison d'avoir le baptême en horreur, et celui qui l'avait conféré ${ }^{42}$ ? Il n'était pas surprenant alors de voir les sauvages cacher leurs enfants pour qu'ils ne soient pas baptisés, rite, semblait-il qui causait la mort, de les voir se boucher les oreilles d'horreur, comme les Juifs devant les paroles du diacre Etienne ${ }^{43}$, pour ne pas entendre les instructions des Pères ${ }^{44}$. Le Père Ragueneau faillit même perdre la vie dans un incident tragique raconté par Chaumonot: "Un jeune homme entre autres ayant aperçu au col du Père Ragueneau un crucifix au bas duquel il y avoit une tête de mort, le lui arracha en criant que c'étoit le sortilège qui les faisoit mourir. Le Père intrépide dans les dangers faisant instance pour le ravoir, l'autre prit une hache pour lui en fendre la tête. Le Père la vit levée sur sa tête sans pâlir et sans trembler: au lieu de s'unfuir et de se mettre en défence il ôta son chapeau, presenta sa tête et attendit le coup. Il l'aurait reçu effectivement si une femme qui se trouva là ne se fut jettée sur la hache au moment qu'il l'abattoit sur le Père ${ }^{45}$."

Une autre fois, le Père Ragueneau pénétra dans une cabane pour baptiser une pauvre malade. Mais le mari de celle-ci entra brusquement en s'écriant: "Jamais ie ne permettray que ma femme soit baptisée, je déteste la Foy, et ie maudis le Dieu des croyans: sors d'icy et ne parle plus". A l'objection du Père... il se saisit d'un gros bâton, à défaut d'une hache qu'il ne trouve pas et décharge sa colère sur le Père. A chaque réponse de celui-ci, il redouble ses coups, et bien que son bâton se brisât en deux après cinq ou six volées, il continue avec ce qui lui reste en mains ${ }^{46}$. Il aurait pu alors répéter avec saint Paul: "Je me réjouis dans mes souffrances pour vous... et dût mon sang être répandu pour servir de sacrifice pour votre foi, je m'en réjouirais ${ }^{47}$."

Chaumonot nous a laissé également un léger écho des souffrances que Ragueneau et lui-même endurèrent en cet hiver: "La nuit

42. Q, 1 (1640): 81.

43. Actes, VII, 57.

44. $\mathrm{AB}, 1: 51$.

45. $\mathrm{AB}, 1:$ 51-52. Le même fait est racontê par le Père Ragueneau lui-même dans $Q, 1$ (1640): 79.

46. Q, 1 (1640): 89.

47. Col, 1, 24. 
nous étoit encore plus fâcheuse que le jour, à cause du froid que nous endurions, étant éloignés du feu et exposés à tout les vents. L'unique soulagement que nous nous procurions étoit en nous couchant sur une écorce d'arbre qui nous servoit de lit, étoit, dis-je de nous mettre les pieds sous les aisselles l'un de l'autre pour les échauffer. Je n'en finirois point $\mathrm{s} 1$ je racontois tous les mauvais traitements qu'on nous fit pour nous obliger à quitter notre mission" ${ }^{48}$. Nos deux missionnaires pouvaient s'approprier ces paroles sublimes de saint Paul: “...persécutés, mais non délaissés; abattus mais non perdus; portant toujours avec nous dans notre corps la mort de Jésus, afin que la vie de Jésus soit aussi manifestée dans notre corps. Car nous qui vivons, nous sommes sans cesse livrés à la mort à cause de Jésus, afin que la vie de Jésus soit aussi manifestée dans notre chair mortelle. ${ }^{49}$ "

Toutefois, le Maître de la moisson réserve à ses fidèles ouvriers quelques consolations: Un jour que Ragueneau et Chaumonot étaient dans la rue, un enfant de quatre ans, plein de santé, s'approche des deux missionnaires et les caresse chaleureusement. Le Père Ragueneau, prévoyant le danger que court cet enfant, au milieu de tant de malades, prie le Père Chaumonot de le baptiser secrètement. Celuici fait fondre de la neige dans ses mains et verse l'eau sur la tête du petit, qui sourit ingénument; il retourne à sa cabane où il tombe malade presqu'aussitôt; ses parents reviennent à la santé, tandis que lui emporté par la maladie, s'envole au Ciel...

Une autre fois, un nouveau-né est attaqué par la petite vérole. Le Père Ragueneau veut bien le baptiser, mais ses parents s'y opposent et d'ailleurs il n'a pas d'eau. Mais voilà que les parents apportent un plat d'eau tiède pour laver le petit. Le Père Ragueneau, avec entrain, se saisit de l'enfant, le plonge et le replonge dans l'eau, le baptisant ainsi tout à son aise, usque ad triam immersionem; après quelques jours, le petit meurt, sans que ses parents sachent qu'il y a un ange de plus au Paradis ${ }^{50} \ldots$

"Que les larmes et les sueurs du pauvre missionnaire, écrit Chaumonot, sont bien essuyées! Que ses fatigues et ses souffrances

48. $\mathrm{AB}, 1: 52$.

49. 11 Cor., IV, 9-11.

50. Q, 1 (1640): 80-81. 
lui donnent de joie lorsqu'il apprend qu'il a ainsi coopéré au salut d'une seule âme, pour l'amour de laquelle le vrai Fils de Dieu n'a pas dédaigné de descendre du Ciel en terre, de se faire homme... en un mot, de donner son sang jusqu'à la dernière goutte, et de mourir en croix $^{51}$."

Quel réconfort aussi pour les missionnaires, quand ils rencontrent des familles chrétiennes comme celle de Joseph Chihouatenhoua, "la perle des chrétiens" 52 , dont la vie pure et charitable rappelle celle des chrétiens de la primitive Eglise ${ }^{53}$.

Ce Joseph Chihouatenhoua mourut tragiquement, le corps transpercé d'une longue épée, frappé de deux coups de hache et scalpé par les Iroquois, alors qu'il travaillait dans son champ ${ }^{54}$. Lourde perte pour les missionnaires, mais épreuve acceptée surnaturellement, comme nous le voyons par le commentaire du Père Lalemant: "Il est vray que nous espérions beaucoup de luy pour la conuersion de ces peuples, dont il s'estoit rendu Apostre durant le cours de cette année; mais puisque les Saincts ont plus de pouvoir lorsqu'ils sont dans le ciel qu'icy bas sur terre, nous deuons croirt que nous auons plus gaigné que perdu à sa mort ${ }^{55}$."

La première étape était accomplie. Etape txtrêmtment dure, harassante, que l'on pourrait résumer par ces paroles aussi pittoresques que réelles du Père Lalemant:

...mais euntes ibant et flebant mittentes semina sua, cette riche moisson ne se fait qu'en arrosant ces terres de sueurs, de larmes et de sang; ie veux dire qu'un missionnaire qui est destiné à ce grand employ, doit se résoudre à mener vn genre de vie bien estonnant, et dans vn dénüement de toutes choses, plus grand qu'on ne peut s'imaginer; à souffrir toutes les iniures de l'air sans soulagement; à endurer mille impertinences, mille brocards et souuent bien des coups de la part des Sauuages Infidèles, incités quelquesfois par les demons, et cela sans consolation humaine; à se trouuer tous les iours dans l'eau ou sur les neiges, sans feu; à passer les mois entiers sans manger

51. Chaumonot à M. Guiotte en 1666 dans Martin, 222.

52. Q, 1 (1640): 85.

53. Voir le récit admirable de la vie de cette famille dans Q, 1 (1640): 85-90.

54. Q, 1 (1640): 102-103.

55. Q, 1 (1640): 103. 
autre chose que du cuir boüilly ou de la mousse qui croist sur les rochers; à trauailler infatigablement, et comme si on auoit un corps de bronze, viure sans nourriture, coucher sans lict, dormir peu, courrir beaucoup, et parmi tout cela auoir la teste preste à receuoir le coup de hache plus souvent que tous les iours, lorsqu'il en prendra fantaisie à un jongleur, ou à quelque mécontent. Bref, il faut estre barbare auec ces Barbares et dire auec l'Apostre: Graecis ac Barbaris debitor sum, faire le Sauuage auec eux et cesser quasi de viure en homme, pour les faire viure en Chrestiens ${ }^{56}$.

Entre cette première mission avec le Père Ragueneau et son départ pour la nation des Neutres avec le Père de Brébeuf, le 2 novembre 1640, Chaumonot fit un court séjour chez les "Arendaenronnons", alors sous la direction du Père Daniel ${ }^{57}$. D'après la Relation de 1640 et l'A utobiographie, ce nouveau poste lui aurait été confié au début du printemps de 1640, probablement en mars.

Chaumonot était tnvoyé auprès de ces sauvages, dont nous aurons à parler plus tard, pour apprendre la langue huronne. Le Père Daniel lui donna ce conseil: "Il vous faut aller tous les jours dans un certain nombre de cabanes pour demander aux sauvages les mots de leur langue et pour les écrire lorsqu'on vous le suggèrera". Et Chaumonot d'ajouter: "J'avois tant de répugnance à faire ces visites qu'à chaque fois que j'entrois dans les cabanes, il me sembloit que j'allois au supplice: tant j'appréhendois les ra lleries qu'il m'y falloit souffrir" ${ }^{58}$. Il ne s'agissait pas de dédain ou de sensibilité excessive, car, au dire du Père Lalemant: "C'était "une petite image de l'enfer" n'y voyant pour l'ordinaire que feu et fumée, et des corps nuds deça et delà noirs et à demy rostis, peslemeslez auec les chiens, qui sont aussi cheris que les enfants de la maison, et dans vne communauté de lict, de plat et de nourriture auec leurs maistres. Tout y est dans la poussière, et si vous entrez

56. Q, 3 (1660): 29-30.

57. Le Père Daniel est né à Dieppe, le 27 mai 1601, et entra au noviciat de Rouen, le ler octobre. Arrivé au Cap-Breton en 1632 et à Québec le 24 juin 1633, il mourut le 4 juillet 1648 à Saint-Joseph II, martyrisé par les Iroquois. Melançon, 23.

58. AB, 1: 52-53. Marie de l'Incarnation écrira: "Nous faisons nos études en cette langue barbare comme font ces jeunes enfants qui vont au collège pour apprendre le latin. Nos Révérends Pères, quoique grands docteurs, en viennent là aussi bien que nous, et ils le font avec une affection et une docilité incroyables", ES, 3: 191. 
dedans, vous ne serez pas au bout de la cabane, que vous serez tout couuert de noirceur, de suye, d'ordure et de pauuretét ${ }^{59}$."

C'est dans de tels milieux que les missionnaires devaient apprendre la langue huronne, selon Chaumonot, "la plus difficile de toutes celles de l'Amérique Septentrionale" "60. Pour apprendre cette langue, écrit Bressani, il faut "outre la grâce de la vocation, un travail opiniâtre". Etudier une langue qui n'a aucune ressemblance avec les langues européennes, à un âge mûr, sans maîtres, sans livres et sans règles, c'est, il faut l'avouer, un travail ardu. La langue huronne était composée en grande partie de voyelles et elle possédait des nombres, des personnes, des inflexions plus nombreuses et plus irrégulières que les langues d'Europe. A cela, il faut ajouter une prononciation étrange, des combinaisons de lettres, des accents, des aspirations, des changements de ton ${ }^{61}$. En 1640, le travail était un peu simplifié, grâce aux études de Jean de Brébeuf, qui n'avait pas craint à quarante ans "de se soumettre pendant trois ans, aux plus cruelles humiliations, au milieu de la cendre et de la fumée des cabanes, pour trouver ce trésor ${ }^{62}$."

Ces difficultés ne devaient pas faire reculer Chaumonot. Au contraire, tout en implorant l'aide du Ciel, il se mit si bien à la tâche qu'il pourra écrire plus tard: "Il plut à Dieu de donner à mon travail, tant de bénédiction, qu'il y a dans le Huron ni tour, ni subtilité, ni manière de s'énoncer dont je n'ai la connoissance et fait pour ainsi dire la découverte. Peut être que Notre Seigneur a voulu récompenser par ce don de langue l'attrait qu'il me donna à l'humilité dès mon noviciat. Peut-être aussi que St. Jérôme à qui j'ai eu recours pendant plusieurs mois m'a assisté dans cet ouvrage ${ }^{63}$ ". Marie de l'Incarnation et le Père Lalemant s'accordent pour confirmer ces paroles: "Il a quasi appris miraculeusement la langue huronne ${ }^{64 "}$;

59. Q, 1 (1639): 57.

60. $\mathrm{AB}, 1: 60$.

61. RA, 85 .

62. RA, 86. Voir aussi Latourelle, 1: 183-188, où l'auteur résume les difficultés rencontrées par les missionnaires pour apprendre la langue et où il montre que la première nécessité pour l'évangélisation des sauvages est la connaissance de la langue et aussi voir: Léon Pouliot, Étude sur les Relations des Jésuites de la Nouvelle-France (1632-1672) (Montréal, 1940), 158-165.

63. $\mathrm{AB}, 1: 60-61$.

64. ES, 3: 230. 
..."Ce fut le Père Chaumonot, que l'on avait reconnu très propre pour les langues" ${ }^{65}$. Après la mort du Père Charles Garnier, tué par les Iroquois en 1649, Chaumonot offrit tous les mérites qu'il pouvait acquérir pendant huit jours pour le repos de l'âme du martyr, à condition "qu'il me feroit son héritier dans la connoissance parfaite qu'il avait du Huron ${ }^{66}$."

Sa prière fut exaucée. "Lorsque je fus envoyé aux Iroquois (que je n'entendois pas) il ne me fallut qu'un mois à apprendre leur langue. J'avoue que souvent j'ai remarqué dans les conseils de leur cinq nations assemblées, que par une assistance de Dieu toute spéciale, je les entendois tous, quoique je n'eusse encore étudié que l'Onnontaguée ${ }^{67}$."

Travail, en tout cas, très profitable car après sa mort on écrira: "Tous les Jésuites qui apprendront jamais le Huron, l'apprendront à la faveur des préceptes, des racines, des discours et de plusieurs autres beaux ouvrages qu'il nous a laissés en cette langue. Les sauvages eux-mêmes avouaient qu'il la parlait mieux qu'eux, qui se piquaient la plupart de bien parler, et qui parlent en effet avec beaucoup de pureté, d'éloquence et de facilité" ${ }^{68}$. Le Père Sébastien Rasle écrira lui-même, en 1723, dans une lettre à son frère: "Le Père Chaumont (sic) qui a demeuré cinquante ans parmi les Hurons, en a composé une grammaire, qui est fort utile à ceux qui arrivent nouvellement dans cette Mission ${ }^{69}$."

Chaumonot donne ses impressions au Père Nappi, dans une lettre de 1640. Elle résume très bien ce chapitre:

L'hiver dernier, il n'y a pas eu une seule cabane dans nos trente-deux bourgs, où la parole de Dieu n'ait pas été portée; mais les fruits ont été plus grands pour l'Eglise triomphante que pour l'Église militante. Comme il régnait une maladie contagieuse qui n'épargnait ni âge, ni sexe, tout notre soin était de catéchiser les malades, pour leur donner

65. Q, 1 (1641): 71 .

66. $\mathrm{AB}, 1: 61$.

67. $\mathrm{AB}, 1: 61$.

68. $\mathrm{AB}, 2: 15$. Ce texte est reproduit aussi dans Rochemonteix, 1: 402 .

69. Sébastien Rasle à son frère, le 12 octobre 1723, JR, 67: 146. Ces deux derniers textes ont le même auteur, si la Suite de la vie du Père Chaumonot a été écrite par le Père Sébastien Rasle. Le rapprochement de ces deux textes pourrait être une preuve en faveur de cette opinion. 
à la fin de leur vie un passe-port pour le ciel. Le plus grand nombre de ceux que cette maladie a enlevés, après le saint baptême, étaient les petits enfants...

Des Sauvages ont tenu plusieurs assemblées très-nombreuses pour aviser aux moyens de nous forcer à quitter le pays. Beaucoup de capitaines ont voté notre mort; mais pas un n'a osé s'en faire l'exécuteur, et jusqu'à présent Dieu nous a préservés de leurs coups. Pendant tout l'hiver nous nous attendions chaque jour à apprendre la mort de quelqu'un de nos missionnaires, et chaque jour, en disant la sainte messe, nous faisions la communion, comme devant nous servir de viatique. Tout s'est borné à quelques coups de bâton, et au chagrin de voir renverser les croix que nous avions dressées, et réduire en cendres une de nos cabanes. Un seul des nôtres a vu couler son sang, sed non usque ad mortem.... ${ }^{70}$

Quatorze mois se sont donc écoulés depuis l'arrivée de Chaumonot au pays des Hurons. Il a été jeté dans la mêlée. Il connaît maintenant ses sauvages; il connaît leurs mœurs, parce qu'il a vécu avec eux; il connaît leur langue, parce qu'il s'est joint à eux, parce qu'il leur a parlé. Son expérience, dans le temps est petite; mais elle est déjà grande par sa connaissance des sauvages. Il est maintenant prêt pour les grandes missions.

\section{André Surprenant, s.j.}

(à suivre)

70. Chaumonot à Nappi, le 26 mai 1640, Carayon, 124-125.

Articles ì paraître dans les Prochaines livraisons:

Wilfrid-H. Paradis, Le nationalisme canadien dans le domaine religieux. L'affaire de l'abbé Thavenet, 1763-1844.

Claude de Bonnault, Le Canada et la conclusion du Pacte de famille en 1761. Gustave Lanctot, A propos du voyage de Jacques Cartier en 1524.

Marcel Trudel, L'Eglise du Canada sous le Régime militaire.

Robert-Lionel Séguin, Les bêtes à corne et leurs implications historiques en Amérique française (2e tranche).

Frère Marcel-Joseph, s.g., et André Surprenant, s.j. Fin de leurs études déjà parues en nos livraisons précédentes. 